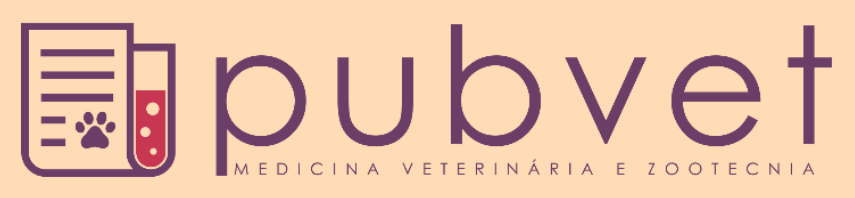

https://doi.org/10.31533/pubvet.v12n11a209.1-8

\title{
Contaminação por fungos filamentosos em sementes de girassol (Helianthus annuus) utilizados na alimentação de psitacídeos
}

\author{
Mariana de Araujo Oliveira' ${ }^{1}$, Maria Carolina Gonçalves Pita $^{2 *} \bullet$ \\ ${ }^{1}$ Discente da Universidade Anhembi Morumbi SP - Brasil, vinculada ao PIC - AC/AM - Programa de Iniciação cientifica - Atividade \\ Complementar Anhembi Morumbi.Email: marianadearaujo.oliveira@hotmail.com \\ ${ }^{2}$ Doutora docente da Universidade Anhembi Morumbi - São Paulo/ SP Brasil. E-mail: mcpita@anhembi.br
}

\begin{abstract}
RESUMO. A demanda por sementes de girassol (Helianthus annuus) vem aumentando devido ao consumo humano e animal, por sua utilização como matéria prima para a produção de biocombustível. O fornecimento dessas sementes na alimentação dos psitacídeos é muito comum, porém é prejudicial à saúde e à longevidade das aves, sendo que uma das causas é a grande contaminação por fungos do gênero Aspergillus e suas toxinas. O quadro de Aspergilose pode se apresentar na forma aguda, que exibe rápida progressão, geralmente culminando com morte súbita da ave, ou crônica, apresentando manifestações clínicas respiratórias e problemas locomotores. Além disso, a presença de aflatoxinas, que são imunossupressoras e cancerígenas, pode levar a manifestações clínicas, dependendo da quantidade ingerida e do tempo de exposição. por meio deste experimento, foi possível demonstrar a alta incidência de contaminação por fungos filamentosos, principalmente por Aspergillus spp., nas sementes de girassol de diferentes origens destinadas à alimentação de aves.
\end{abstract}

Palavras-chave: aspergillus, aves, curvularia, penicillium, rhizopus

\section{Contamination by filamentous fungi in sunflower seeds (Helianthus annuus) used for feeding psittacines}

\begin{abstract}
The demand by sunflower seeds (Helianthus annuus) have been incresing due to human and animal consumption, as well as their raw material utilization for the production of biofuel. The supply of these seeds in psittacines feeding is very common, but it is detrimental to the health and longevity of these birds. The large contamination by genus Aspergillus fungi and its toxins figures as one of the causes of this damage. Aspergillosis can present in the acute form that exhibits rapid progression, usually culminating with sudden death of the bird, or chronic, presenting respiratory clinical manifestations and locomotion problems. In addition, the presence of aflatoxins, which are immunosuppressive and carcinogenic, may lead to clinical manifestations depending on the amount and the time of exposure. Through this experiment, it was possible to demonstrate the high incidence of contamination by filamentous fungi, mainly by Aspergillus spp., in sunflower seeds of different origins, destined to feed birds.
\end{abstract}

Keywords: aspergillus, birds, curvularia, penicillium, rhizophus

\section{Contaminación por hongos filamentosos em semillas de girasol (Helianthus annuus) utilizados em la alimentación de lós psitacídeos}


RESUMEN. Las semillas de girasol (Helianthus annuus) vienen aumentando su demanda debido al consumo humano y animal, así como materia prima para la producción de biocombustible. Entre lós psitacídeos, el suministro de estas semillas en la alimentación es muy común, pero es perjudicial para la salud y longevidad de las aves. El cuadro de Aspergilosis puede presentarse en la forma aguda que muestra una rápida progresión, generalmente culminando con muerte súbita del ave, o crónica, presentando manifestaciones clínicas respiratorias y problemas locomotores. Además, la presencia de aflatoxinas, que son inmunosupresoras y cancerígenas, puede llevar a manifestaciones clínicas dependiendo de la cantidad ingerida y el tiempo de exposición. A través de este experimento, fue posible demostrar la alta incidência de contaminación por hongos filamentosos, principalmente por Aspergillus spp., en las semillas de girasol de diferentes orígenes, destinadas a la alimentación de aves.

Palabras clave: aspergillus, aves, curvularia, penicillium, rhizopus

\section{Introdução}

Grande parte das aves criadas em cativeiro, especialmente psitacídeos, é alimentada apenas com sementes de girassol ou tem a maior parte da sua dieta baseada nessa semente. Embora tais sementes sejam muito apreciadas pelas aves, por sua palatabilidade, são extremamente prejudiciais à saúde e à longevidade das aves (Godoy, 2006; Maia \& Siqueira, 2007) por apresentarem altos níveis de energia, ausência ou baixa concentração das vitaminas $\mathrm{A}, \mathrm{D} 3$, e do complexo $\mathrm{B}$, deficiência de inúmeros minerais, tal como cálcio, desproporção na relação entre cálcio e fósforo, além de apresentar altas concentrações de lipídios e grande contaminação pelos fungos Aspergillus e suas toxinas (Maia \& Siqueira, 2007; Conceição et al., 2010), relacionados à inadequação de "Boas práticas de Fabricação" e dos processos finais de industrialização das sementes (Forsythe, 2002).

A suscetibilidade das aves a essa micose é proporcionada, principalmente, pela característica do seu trato respiratório, com ausência de diafragma e presença de sacos aéreos, que contribuem para um excelente local de colonização fúngica, com ótimas condições de temperatura e oxigênio e pouca vascularização (Tell, 2005).

As micotoxicoses são causadas por metabólicos tóxicos secundários de fungos que, em geral, interferem na resistência imunológica das aves. A presença das aflatoxinas, que são imunossupressoras e cancerígenas (Wood, 1992), pode levar a manifestações clínicas, dependendo da quantidade ingerida e do tempo de exposição.

O presente trabalho teve como objetivo analisar a contaminação de sementes de girassol de diferentes origens por fungos filamentosos, vendidas comercialmente em São Paulo - SP e destinadas à alimentação de aves.

\section{Material e métodos}

$\mathrm{O}$ presente experimento foi realizado nos Laboratórios de Microbiologia e Patologia Clínica da Universidade Anhembi Morumbi - São Paulo - SP, no período de Abril a Dezembro de 2017. Foram utilizadas vinte amostras de sementes de girassol, classificadas da seguinte maneira: de 1 a 10 sementes envasadas de diferentes marcas comerciais; e, de 11 a 20 , sementes vendidas a granel em diversos estabelecimentos, comumente comercializadas na cidade de São Paulo/ SP Brasil.

Os grãos foram triturados e homogeneizados (Forsythe, 2002), pesados $10 \mathrm{~g}$ e diluídos em 100 $\mathrm{ml}$ de água peptonada a $0,1 \%$. Foram preparadas diluições da amostra SND (sem nenhuma diluição) de $10^{-1}$ a $10^{-4}$ pelas sucessivas transferências de $1,0 \mathrm{ml}$ em suspensão e inoculadas em meio seletivo, "Agar Saboraud Dextrose" - KASVI. As amostras foram avaliadas no período de 72 e 168 horas de incubação a uma temperatura de $22^{\circ} \mathrm{C}$.

As colônias viáveis foram isoladas e inoculadas em microcultivo em uma lâmina com lamínula, para promover o crescimento reprodutivo, e incubadas a uma temperatura de $22^{\circ}$ C por 168 horas. A leitura ocorreu por meio da preparação da lâmina e da observação em azul de algodão de lactofenol, utilizando um microscópio com aumento de 40 e 100x.

\section{Resultados}

As amostras de 1 a 7 apresentaram resultados positivos para fungos filamentosos. Os fungos isolados foram: A. niger, em quatro amostras; $A$. flavus; A. fumigatus; A. glaucus; Curvularia sp.; e Penicillium spp., em uma amostra cada. O fungo isolado no maior número de amostras foi $\mathrm{o}$ Aspergillus spp. (Tabela 1). 
Em relação aos Aspergillus spp., foram isoladas quatro espécies, sendo o A. niger (Figura 1-2) o mais frequente, seguido por A. flavus (Figura 3-5), A. fumigatus (Figura 3-6) e A. glaucus (Figura 1-1). As amostras 2 e 7 apresentaram duas espécies de Aspergillus. $\mathrm{Na}$ amostra 6 isolou-se o A. niger (Figura 1-2) e Curvularia sp. (Figura 2-3), enquanto na amostra 5 observou-se o isolamento apenas de fungo do gênero Penicillium spp. (Figura 2-4) (Tabela 1).

As amostras de girassol analisadas numeradas de 11 a 20 (vendidas a granel) apresentaram resultados positivos para fungos filamentosos, sendo que o Aspergillus e o Rhizopus apresentaram maior quantidade, de dez e seis amostras contaminadas, respectivamente (Tabela 2).

Em relação aos Aspergillus spp., foram isoladas três espécies, sendo o A. niger (Figura 12) o mais frequente, seguido por A. flavus (igura 3-5) e A. fumigatus (Figura 3-6), isolados duas e uma vez, respectivamente, durante o período de análise. A amostra 15 apresentou duas espécies de Aspergillus e a amostra 14 apresentou três espécies de Aspergillus. As amostras 11, 12, 13, 16, 17 e 19 apresentaram o isolamento de A. niger (Figura 1-2) e de Rhizopus sp. (Figura 4-7) (Tabela 2).

Com relação aos números de diluições realizadas, as amostras de sementes de girassol de números $1,8,9$ e 10 (envasadas e de marcas comerciais) não apresentaram nenhum gênero de fungos filamentosos isolados, em nenhuma diluição. $\mathrm{O}$ isolamento de fungos filamentosos nas diluições de $10^{-1}$ a $10^{-2}$ foi relatado em quatro amostras. Duas amostras apresentaram isolamento apenas na diluição de $10^{-1}$ e apenas uma amostra apresentou isolamento nas diluições de $10^{-1}$ a $10^{-4}$ (Tabela 3). Aspergillus, Curvularia e o Penicillium estão presentes nas diluições de quatro, uma e duas amostras analisadas, respetivamente ( $\underline{\text { Tabela } 3})$.

Tabela 1. Isolamento de espécies e gêneros de fungos filamentosos em amostras de sementes de girassol de marcas comerciais, independente das diluições, avaliadas no período de Abril a Dezembro de 2017.

\begin{tabular}{|c|c|c|c|c|c|c|c|}
\hline \multirow[t]{2}{*}{ Amostras } & \multicolumn{7}{|c|}{------------- Espécies e gêneros de fungos filamentosos isolados--------------- } \\
\hline & A. niger & A. flavus & A. fumigatus & A. glaucus & Rhizopus sp. & Curvularia sp. & Penicillium spp. \\
\hline Amostra 1 & I & NI & NI & NI & NI & NI & NI \\
\hline Amostra 2 & NI & I & I & NI & NI & NI & NI \\
\hline Amostra 3 & NI & NI & NI & NI & NI & NI & I \\
\hline Amostra 4 & I & NI & NI & NI & NI & NI & NI \\
\hline Amostra 5 & NI & NI & NI & NI & NI & NI & I \\
\hline Amostra 6 & I & NI & NI & NI & NI & I & NI \\
\hline Amostra 7 & I & NI & NI & I & NI & NI & NI \\
\hline Amostra 8 & NI & NI & NI & NI & NI & NI & NI \\
\hline Amostra 9 & NI & NI & NI & NI & NI & NI & NI \\
\hline Amostra 10 & NI & NI & NI & NI & NI & NI & NI \\
\hline
\end{tabular}

NI - não isolado, I - isolado. Fonte: arquivo pessoal (2017).

Tabela 2. Isolamento de espécies e gêneros de fungos filamentosos em amostras de sementes de girassol vendidas a granel, independente das diluições, avaliadas no período de Abril a Dezembro de 2017.

\begin{tabular}{|c|c|c|c|c|c|c|c|}
\hline Amostras & ---- & ----- Espéc & es e gêneros de & ungos filame & tosos isolados & ------------- & \\
\hline & A. niger & A. flavus & A. fumigatus & A. glaucus & Rhizopus sp. & Curvularia sp. & Penicillium spp. \\
\hline Amostra 11 & I & NI & NI & NI & I & NI & NI \\
\hline Amostra 12 & I & NI & NI & NI & I & NI & NI \\
\hline Amostra 13 & I & NI & NI & NI & I & NI & NI \\
\hline Amostra 14 & I & I & I & NI & NI & NI & NI \\
\hline Amostra 15 & I & I & NI & NI & NI & NI & NI \\
\hline Amostra 16 & I & NI & NI & NI & I & NI & NI \\
\hline Amostra 17 & I & NI & NI & NI & I & NI & NI \\
\hline Amostra 18 & I & NI & NI & NI & NI & NI & NI \\
\hline Amostra 19 & I & NI & NI & NI & I & NI & NI \\
\hline Amostra 20 & I & NI & NI & NI & NI & NI & NI \\
\hline
\end{tabular}

NI - não isolado, I - isolado. Fonte: arquivo pessoal (2017). 
Tabela 3. Isolamento de espécies e gêneros de fungos filamentosos em amostras de sementes de girassol de marcas comerciais, em diferentes números de diluições, avaliadas no período de Abril a Dezembro de 2017.

\begin{tabular}{|c|c|c|c|c|}
\hline \multirow[t]{2}{*}{ Amostras } & \multicolumn{4}{|c|}{ 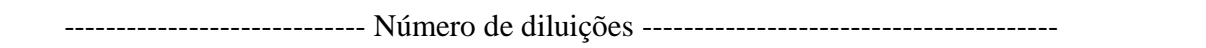 } \\
\hline & $10^{-1}$ & $10^{-2}$ & $10^{-3}$ & $10^{-4}$ \\
\hline Amostra 1 & NI & NI & NI & NI \\
\hline \multirow[t]{2}{*}{ Amostra 2} & A. flavus & A. flavus & A. flavus & A. flavus \\
\hline & A. fumigatus & A. fumigatus & & \\
\hline Amostra 3 & Penicillium spp. & Penicillium spp. & NI & NI \\
\hline Amostra 4 & A. niger & NI & NI & NI \\
\hline Amostra 5 & Penicillium spp. & Penicillium spp. & NI & NI \\
\hline \multirow[t]{2}{*}{ Amostra 6} & A. niger & A. niger & NI & NI \\
\hline & Curvularia sp. & Curvularia sp. & & \\
\hline Amostra 7 & A. niger & NI & NI & NI \\
\hline Amostra 8 & NI & NI & NI & NI \\
\hline Amostra 9 & NI & NI & NI & NI \\
\hline Amostra 10 & NI & NI & NI & NI \\
\hline
\end{tabular}

NI - não isolado. Fonte: arquivo pessoal (2017).

$\mathrm{O}$ isolamento de fungos filamentosos nas diluições de $10^{-1}$ a $10^{-4}$ foi relatado em cinco amostras de sementes de girassol vendidas a granel. Observou-se isolamento em quatro amostras nas diluições de $10^{-1}$ a $10^{-2}$ e apenas em uma amostra nas diluições de $10^{-1}$ a $10^{-3}$ (Tabela 4), sendo que o Aspergillus e o Rhizopus foram isolados no maior número de diluições (Tabela 4).

Tabela 4. Isolamento de espécies e gêneros de fungos filamentosos em amostras de sementes de girassol vendidas a granel, em diferentes números de diluições, avaliadas no período de Abril a Dezembro de 2017.

\begin{tabular}{|c|c|c|c|c|}
\hline \multirow[t]{2}{*}{ Amostras } & \multicolumn{4}{|c|}{--- } \\
\hline & $10^{-1}$ & $10^{-2}$ & $10^{-3}$ & $10^{-4}$ \\
\hline \multirow[t]{2}{*}{ Amostra 11} & A. niger & A. niger & A. niger & NI \\
\hline & Rizhopus sp. & & & \\
\hline \multirow[t]{2}{*}{ Amostra 12} & A. niger & A. niger & NI & NI \\
\hline & Rhizopus sp. & & & \\
\hline Amostra 13 & Rizhopus sp. & Rhizopus sp. & Rhizopus sp. & Rhizopus sp. \\
\hline \multirow[t]{3}{*}{ Amostra 14} & A. niger & A. niger & NI & NI \\
\hline & A. flavus & & & \\
\hline & A. fumigates & & & \\
\hline \multirow[t]{2}{*}{ Amostra 15} & A. niger & A. niger & A. niger & A. niger \\
\hline & A. flavus & A. flavus & A. flavus & \\
\hline \multirow[t]{2}{*}{ Amostra 16} & A. niger & A. niger & A. niger & A. niger \\
\hline & Rhizopus sp. & Rhizopus sp. & Rhizopus sp. & Rhizopus sp. \\
\hline \multirow[t]{2}{*}{ Amostra 17} & A. niger & A. niger & A. niger & A. niger \\
\hline & Rhizopus sp. & Rhizopus sp. & Rhizopus sp. & \\
\hline Amostra 18 & A. niger & A. niger & A. niger & A. Níger \\
\hline \multirow[t]{2}{*}{ Amostra 19} & A. niger & A. niger & NI & NI \\
\hline & Rhizopus sp. & & & \\
\hline Amostra 20 & A. niger & A. niger & NI & NI \\
\hline
\end{tabular}

NI - não isolado. Fonte: arquivo pessoal (2017) 


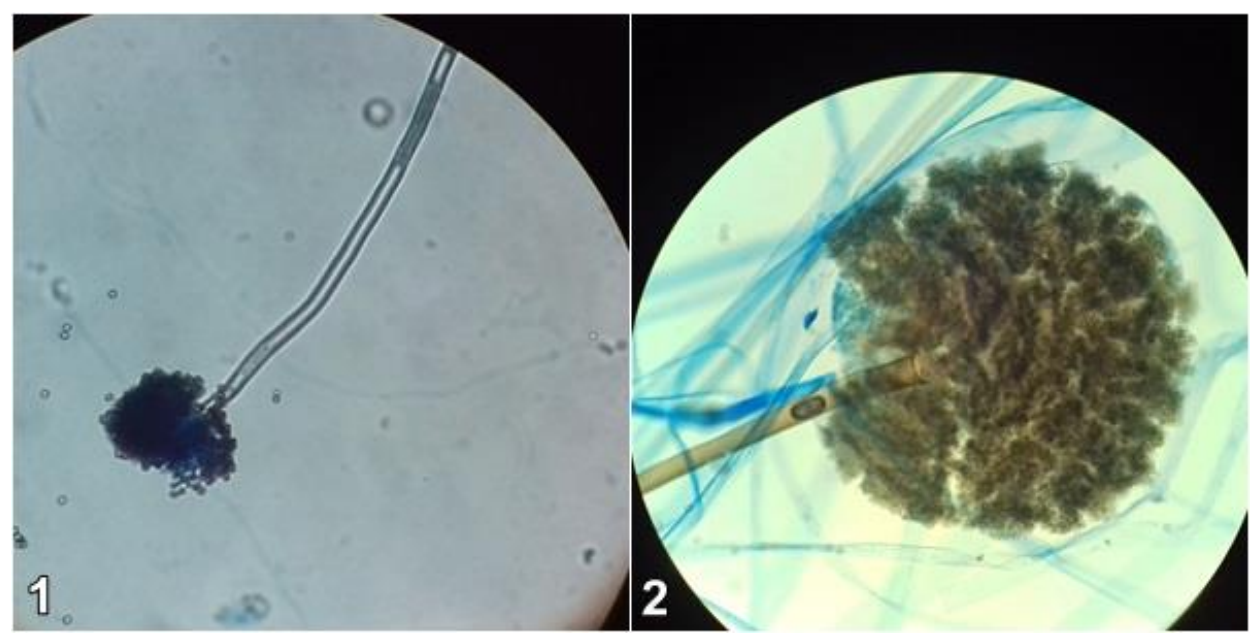

Figura 1. (1) Visualização microscópica de fungo filamentoso Aspergillus glaucus isolado de uma amostra de sementes de girassol de marca comercial. (2) Visualização microscópica de fungo filamentoso Aspergillus niger isolado de uma amostra de sementes de girassol de marca comercial. Fonte: arquivo pessoal, Laboratório de Microbiologia da Universidade Anhembi Morumbi - São Paulo/ SP (2017).

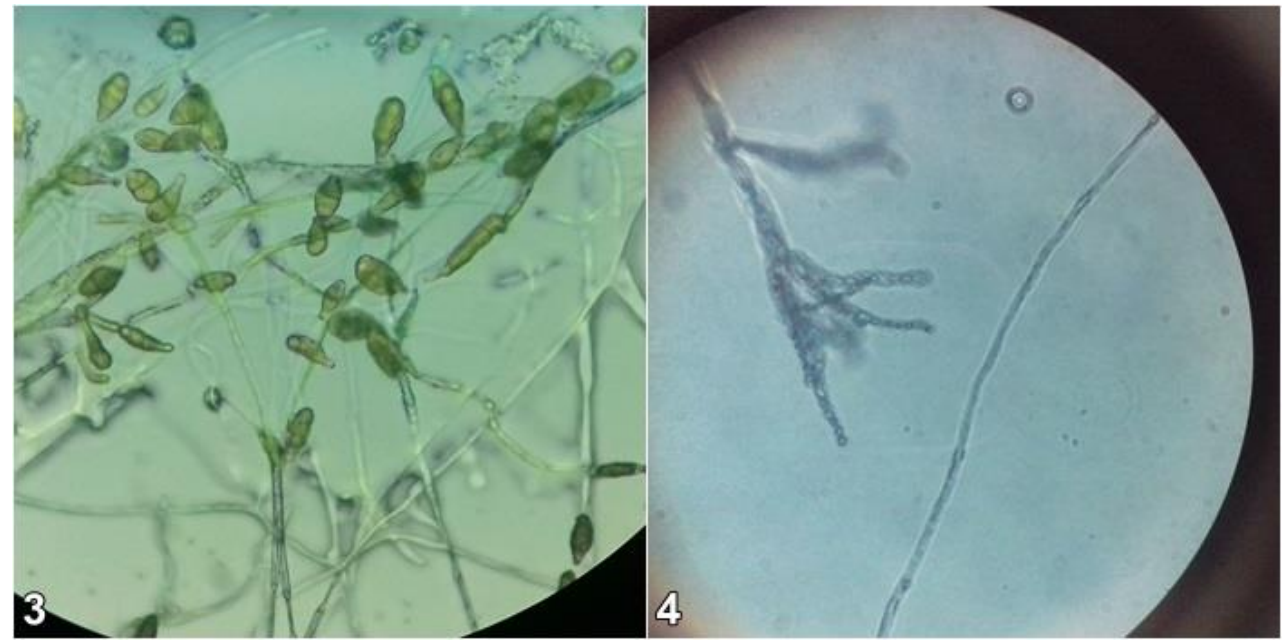

Figura 2. (3) Visualização microscópica de fungo filamentoso Curvularia sp. isolado de uma amostra de sementes de girassol de marca comercial. (4) Visualização microscópica de fungo filamentoso Penicillium spp. isolado de uma amostra de sementes de girassol de marca comercial. Fonte: arquivo pessoal, Laboratório de Microbiologia da Universidade Anhembi Morumbi São Paulo/ SP (2017).

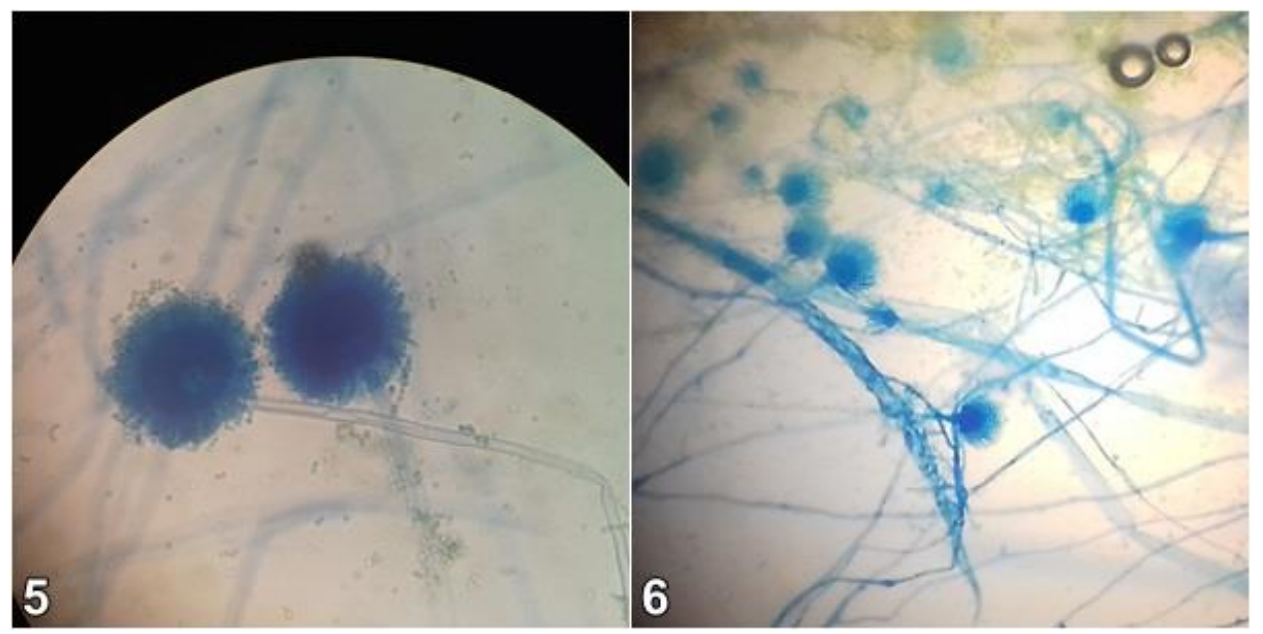

Figura 3. (5) Visualização microscópica de fungo filamentoso Aspergillus flavus isolado de uma amostra de sementes de girassol vendidas a granel. (6) Visualização microscópica de fungo filamentoso Aspergillus fumigatus isolado de uma amostra de sementes de girassol vendidas a granel. Fonte: arquivo pessoal, Laboratório de Microbiologia da Universidade Anhembi Morumbi - São Paulo/ SP (2017). 


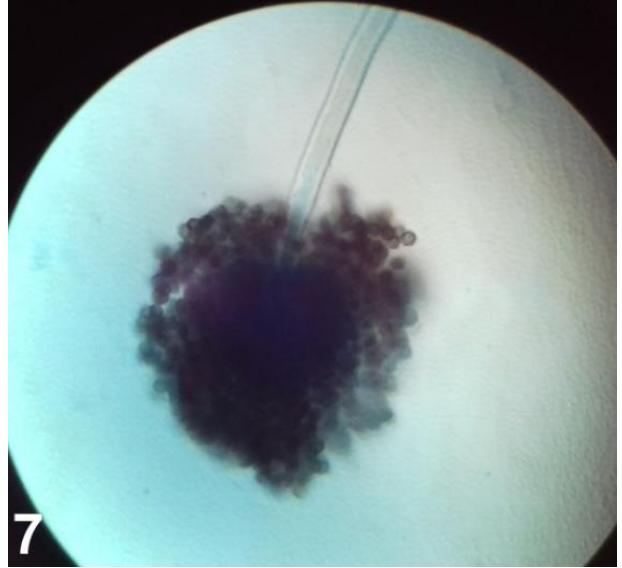

Figura 4. (7) Visualização microscópica de fungo filamentoso Rhizopus sp. isolado de uma amostra de sementes de girassol vendidas a granel. Fonte: arquivo pessoal, Laboratório de Microbiologia da Universidade Anhembi Morumbi - São Paulo/ SP (2017

\section{Discussão}

Foi relatado na pesquisa de Pitt \& Miscamble (1994) o crescimento de A. flavus em amostras de sementes de girassol, independente da origem, em temperaturas de $25^{\circ}, 30^{\circ}$ e $37^{\circ} \mathrm{C}$, e o crescimento de A. flavus e A. fumigatus em sementes de girassol de marcas comerciais e vendidas a granel, à temperatura de $28^{\circ}$ a $30^{\circ} \mathrm{C}$, em 2010 , por Conceição et al. 2010 evidenciando, por meio do presente experimento, o crescimento desses fungos filamentosos também à temperatura de $22^{\circ}$ $\mathrm{C}$, sabendo-se que a maioria das espécies é mesófila e apresenta ótimo crescimento em temperaturas entre $21^{\circ}$ a $35^{\circ} \mathrm{C}$ (Dhingra, 1985).

A aspergilose é causada por fungos do gênero Aspergillus spp. (Revolledo \& Ferreira, 2009) encontrados em terras úmidas, vegetação em decomposição, debris orgânicos e grãos. O quadro pode apresentar-se na forma aguda, principalmente em aves jovens, ocasionada pela exposição e inalação de grande quantidade de esporos, e apresenta rápida progressão, geralmente culminando com a morte súbita da ave (Rupley, 1999; Carciofi \& Oliveira, 2007) e na forma crônica, comum em aves adultas criadas em cativeiro, induzida por condições de estresse, imunossupressão e manejo inadequado, gerando uma queda da resistência imunológica (Vanderheyden, 1993).

Este experimento evidenciou a maior contaminação das sementes de girassol vendidas a granel e expostas ao ambiente de comercialização, em relação às envasadas e de marcas comerciais. Tais resultados estão relacionados à rigorosidade das "Boas Práticas de Fabricação" e ao monitoramento adequado dos processos finais de industrialização (Conceição et al., 2010), além do armazenamento em condições de temperatura, umidade e oxigenação adequados, pois esses fungos crescem em ambientes com pouca ventilação, associados a temperaturas e umidade elevadas, propiciando e facilitando o crescimento do agente (Xavier et al., 2007).

No caso das amostras vendidas a granel, a grande contaminação por fungos filamentosos pode ocorrer durante a colheita, as fases de secagem e o armazenamento da semente, fatores que oferecem um ambiente favorável para o crescimento e reprodução desses fungos, propiciando também, a produção de micotoxinas por estes (Pitt \& Hocking, 1997).

O fungo do gênero Penicillium spp., assim como o Aspergillus, é um fungo de estocagem e produtor de micotoxinas (Lima \& Araujo, 1999), sendo o seu crescimento relacionado aos mesmos fatores do Aspergillus, considerando-se uma temperatura ideal para o seu crescimento, valores entre $21^{\circ} \mathrm{e} 35^{\circ} \mathrm{C}$ e umidade relativa do ar de 80 a 90\% (Dhingra, 1985), porém, este não apresenta importância como agente de doença, exceto quando seu hospedeiro se apresenta debilitado, vivendo na forma saprófita em aves sadias, assim como o fungo do gênero Curvularia sp. (Mourão et al., 2017), sendo esse o agente casual da mancha foliar do milho.

O fungo do gênero Rhizopus sp. ocasiona a redução da germinação e do vigor das sementes (Barreto et al., 2004), relatado tipicamente como fungo de deterioração somente quando as condições de armazenamento forem impróprias à manutenção da qualidade do produto (Gimeno, 2000; Hussein \& Brasel, 2001).

O crescimento do fungo, e consequentemente a produção de micotoxinas, está relacionado com a umidade (atividade de água favorável 0,80>0,99, considerado ótimo $85 \%$ ), a temperatura (entre $8^{\circ} \mathrm{a}$ $42^{\circ} \mathrm{C}$ ), a presença de hidrogênio e a composição do substrato, sendo que a presença do fungo não indica necessariamente a produção da micotoxina (Gourama \& Bullerman, 1995; Tanaka et al., 2001; Diniz, 2002; Pereira et al., 2002), estas normalmente são termoestáveis, portanto, resistem a tratamentos térmicos e seus efeitos no organismo das aves são irreversíveis (Lavor \& Sousa, 2008).

$\mathrm{O}$ controle dos fungos e das micotoxinas pode ser conseguido mantendo-se a baixa umidade dos 
grãos e o alimento fresco, além da limpeza dos equipamentos e do uso de inibidores de crescimento dos fungos (Jones, 2000).

Durante a análise das amostras de sementes, tanto envasadas quanto a granel, observou-se o crescimento de leveduras, no entanto, por não fazerem parte do foco desta pesquisa, elas não foram classificadas. A ênfase deste trabalho diz respeito apenas às colônias de fungos filamentosos.

Por este experimento foi possível demonstrar a alta incidência de contaminação das sementes de girassol destinadas à alimentação de aves por fungos filamentosos, principalmente por Aspergillus spp.Estes resultados poderão ser utilizados como referência para o estudo da clínica de aves e como indicação sobre a alimentação de psitacídeos criados em cativeiro, portanto, sendo fundamental um cuidado especial com a ração, atentando-se para a aquisição de itens de qualidade e que estes sejam armazenados adequadamente para coibir o crescimento de fungos e de suas toxinas.

\section{Referências}

Barreto, A. F., Egberto, A., Bonifácio, B. F., Ferreira, O. R. R. S. \& Belém, L. F. 2004. Qualidade fisiológica e a incidência de fungos em sementes de algodoeiro herbáceo tratadas com estrados de agave. Revista de Oleaginosas e Fibrosas, 8, 2-3, 839-849.

Carciofi, A. C. \& Oliveira, L. D. 2007. Doenças Nutricionais. In: Cubas, Z. S., Silva, J. R. \& Catão-Dias, J. L. Tratado de Animais Selvagens - Medicina Veterinária (1a ed., Vol 1, p. 838-864). Roca, São Paulo, Brasil.

Conceição, A. M., Melo, C. B. \& Sarmento, C. A. P. 2010. Contamination by Aspergillus flavus and A. fumigatus in sunflower seeds used in psittacine bird food. Revista Biotemas, 145-148.

Dhingra, O. D. 1985. Losses caused by microorganisms during storage of seeds. Revista Brasileira de Sementes, 7(1), 139-145.

Diniz, S. P. S. S. 2002. Micotoxinas. Livraria e Editora Rural, Campinas, São Paulo, BR.

Forsythe, S. J. 2002. Microbiologia de Segurança Alimentar. Ed. Artmed, Porto Alegre, BR.

Gimeno, A. 2000. Revision genérica Del problema de los hongos y de lãs micotoxinas en al alimentacion animal. Special Nutrients.

Godoy, S. N. 2006. Psittaciformes. In: Cubas, Z. S., Silva, J. C. R., Catão-Dias J. L. Tratado de
Animais Selvagens Medicina Veterinária. Roca, São Paulo, Brasil.

Gourama, H. \& Bullerman, L. B. 1995. Detection of molds in foods and feeds: potential rapid and selective methods. Journal of Food Protection, 58(12), 1389-1394.

Jones, F. T. 2000. Control of toxine substances. Feedstuffs Reference Issue, 72, 90-94.

Lavor, C. T. B. \& Sousa, F. M. 2008. Ostrich micotoxity Struthio camelus. Case Report. Revista Ciencia Agronómica, 39(2), 348-352.

Lima, E. F. \& Araújo, A. 1999. E. Fungi causing tipping, transported and transmitted through peanut seed. Revista Brasileira de Oleaginosas e Fibrosas, 3(2), 71-76.

Maia, P. P. \& Siqueira, M. E. P. B. 2007. Aflatoxins in pet foods - A Review. Revista da FZVA, 14(1), 235-257.

Mourão, A. S. C., Ságio, S. A., Souza, M. R. \& Santos, G. R. 2017. Morphological and molecular identification of Curvularia $s p$. casual agent of corn leaf stain. Revista Brasileira de Milhoo e Sorgo, 16(1), 1-12.

Pereira, M. M. G., Carvalho, E. P. \& Prado, G. 2002. Growing and production aflatoxins by Aspergillus flavus and Aspergillus parasiticus. Boletim do Centro de Pesquisa de Processamento de Alimentos, 20(1).

Pitt, J. I. \& Hocking, A. D. 1997. Fungi and food spoilage $\left(2^{\text {nd }}\right.$ ed.). Chapman e Hall, London, UK.

Pitt, J. I. \& Miscamble, B. F. 1994. Water relations of Aspergillus flavus and closely related species. Journal of Food Protection, 58(1), 8690.

Revolledo, L. \& Ferreira, A. J. P. 2009. Patologia Aviária - Doenças produzidas por fungos $e$ suas toxinas (p. 342-362). Ed. Manole Ltda., Tamboré, BR.

Rupley, A. E. 1999. Doenças Metabólicas e Doenças Nutricionais. In: Rupley, A. E. Manual de Clinica Aviária. Roca, São Paulo, Brasil.

Tanaka, M. A. S., Maeda, J. A. \& Plazas, I. H. A. Z. 2001. Fungi associated to corn seeds under storage conditions. Sciencia Agricola, 58 (3), 501-508.

Tell, L. A. 2005. Aspergillosis in mammals and birds: impact on veterinary medicine. Medical Mycology, 43(1), 571-573.

Vanderheyden, N. 1993. Aspergillosis in psittacine chicks. In G. Jackson (Ed.). 
Proceedings of the Annual Conference of the Association of Avian Veterinarians (p. 207). Nashville, TN, USA.

Wood, G. E. 1992. Mycotoxins in foods and feeds in the United States. Journal of Animal Science, 70(12), 3941-949.

Xavier, M. O., Soares, M. P., Meinerz, A. R. M., Nobre, M. O., Osório, L. G., da Silva Filho, R. P. \& Meireles, M. A. C. A. 2007. Aspergillosis: a limiting factor during recovery of captive magellanic penguins. Brazilian Journal of Microbiology, 38, 480-488.

Recebido: 23.08 .2018 .

Aprovado:23.09.2018.

Publicado: 12112018

Licenciamento: Este artigo é publicado na modalidade Acesso Aberto sob a licença Creative Commons Atribuição 4.0 (CC-BY 4.0), a qual permite uso irrestrito, distribuição, reprodução em qualquer meio, desde que o autor e a fonte sejam devidamente creditados. 